\title{
Ionosphere-grid-aided fast acquisition algorithm of a satellite FFH telemetry signal
}

\author{
Xiao Chen ${ }^{\dagger}$, \\ 403 Institute, Rocket Force University, Xi'an, Shaanxi,710038, China \\ $\dagger$ E-mail: ty_chenxiao@163.com \\ Li-Yi He, Wei-Zhi Li and Shuai Yu \\ 96177 Troop,PLA, Huangshan,Anhui,245400,China \\ E-mail:625997317@qq.com
}

\begin{abstract}
Coherent Fast Frequency Hopping (FFH) spread spectrum signals effectively improve the ability to fend off forwarding jamming and interception of a satellite anti jamming communication system. However the acquisition of FFH signal is influenced by the frequency-selective ionosphere delays. The global ionosphere grid of Vertical Total Electron Content (VTEC) is used to aid the acquisition.The ionosphere delays were modeled as functions of VTEC and elevation angles, the integrals of different hops were adjusted and accumulated accordingly thus the acquisition complexity was reduced. The energy loss caused by VTEC error is calculated and the complexity reduction of this algorithm versus TEC searching algorithm is analyzed. This research is valuable to the synchronization of satellite FFH signal on different radio bands.
\end{abstract}

Key words: ionosphere dispersion; satellite communications; FFH; signal acquisition; global ionosphere grid; coherent integral

\section{Introduction}

Frequency Hopping Spread Spectrum(FHSS) can enhance the anti-jamming and anti-inception ability of radio signals in wireless communication and is widely used in military satellite communications[1]. According to the existing open information sources, most FHSS communication systems in satellite communication adopt non-coherent and slow FH schemes due to the high reliability requirement and the current development level of electronic devices[2]. In a non-coherent slow FH communication system the symbol rate is usually greater than or equal to the frequency hopping speed and there are at least more than one information symbols in one FH cycle, so when this frequency point is jammed the demodulation bit errors will appear. Comparatively, when a fast FH pattern is used, one information symbol is modulated by $N \mathrm{FH}$ frequencies[3]. This paper deals with the signal acquisition 
problem of a fast FHSS satellite communication signal under ionosphere delay.

Without considering the effects of ionosphere, the signal acquisition of FHSS signal is a two-dimensional searching process, during which the receiver calculates the slide correlations of the local replica signal and the receiving signal while searching through different Doppler values and time-of-arrival values and finds the maximum correlation result. The acquisition time depends on the uncertainty of doppler, the uncertainty of time of arrival and the cycle length of FH pattern, this acquisition period is shortened when time-domain and frequency-domain parallel acquisition methods are adopted[3]. The very important research direction of this paper is the impact of ionosphere on signal acquisition. Being the unavoidable media during satellite-ground telecommunication, the dispersion characteristics of ionosphere introduces different delays, which are up to the different frequencies and the total electron content(TEC) along the transmission path, to the frequency hopping waves in one modulation symbol[4]. Those delays, in return, bring about accumulation losses during signal acquisition and integral calculation, so in this paper we introduce the compensation of TEC in the design of signal acquisition algorithms and constrain the energy loss in the decision of signal acquisition. Ionosphere Grid is a global vertical TEC network that built by GPS dual frequency receivers with the TEC precision of two to three TEC units[5] (one TEC units being $10^{16}$ electrons $/ \mathrm{m}^{2}$ ). Based on the research of TEC compensation in signal acquisition, this paper proposes Ionosphere-Grid-aided fast acquisition algorithm and improves the acquisition performance.

\section{Signal Model}

\subsection{Fast FH signal model in satellite communication}

This paper deals with a phase-coherent fast FH signal, which adopts a binary phase shift keying(BPSK) modulation. The FH patter is block hopping, meaning that the cycle length of frequency hopping equals to that of one modulation symbol, thus both the block length and the cycle of auto-correlation function is $\mathrm{N}$ hops[6]. The signal can be written as

$$
s(t)=\sqrt{2 S} \sum_{i=1}^{N} \operatorname{Re}\left[d(t) e^{j 2 \pi f_{R F} t} e^{j 2 \pi f_{i} t}\right]
$$

whereSis the average transmitting power of the signal, $f_{i}$ is the hopping frequency in the $i$-th hop, $f_{R F}$ is the reference radio frequency and the actual hopping radio frequency is $f_{i}+f_{R F}$. Let $T_{\mathrm{h}}$ be the duration time of one frequency hop and one FH cycle is made up of $N T_{\mathrm{h}} \mathrm{s}, \operatorname{Re}[\cdot]$ indecate the real part and $d(t)$ is the modulation data. To guarantee that the initial carrier phases in consecutive hop periods are the same, the frequency hopping pattern is designed as 


$$
f_{i}=\frac{k}{T_{h}}, k \in\{1,2, \ldots, K\}, i=1,2,3, \ldots, N .
$$

where $k$ indicates the hopping pattern, $f_{\mathrm{i}}$ is the $k$-th harmonic of $1 / T_{\mathrm{h}}$, the radio $\mathrm{FH}$ bandwidth is $B_{\mathrm{H}}=K / T_{\mathrm{h}}$, and $N<<K$. The uplink and downlink signals both adopt this signal model.

\subsection{Auto-correlation function under ionosphere effects}

Signal acquisition is the process of searching the maximum of auto-correlation function in time-of-arrival range and doppler frequency range. the autocorrelation function of the coherent fast FH signal $\mathrm{R}(\mathrm{v}, \tau)$ is defined as the coherent accumulation of several auto-correlations in different frequency hopping periods, its complex-value form can be written as

$$
R(v, \tau)=\sum_{i} R_{i}(v, \tau)
$$

where the auto-correlation function in one $\mathrm{FH}$ period can be expressed as

$$
R_{i}(v, \tau)=\int_{(i-1) T_{h}}^{i T_{h}} e^{-j 2 \pi\left[f_{i} \cdot \tau-\left(f_{R F}+f_{i}\right) \frac{(i-1) T_{h} v}{c}\right]} \mathrm{d} t .
$$

under none-ionosphere assumption, $R(v, \tau)$ is the function of time-of-arrival uncertainty $\tau$ and the satellite-ground-velocity uncertainty $v$ because the doppler frequency is the function of $v$. Since there already exists thorough study on the acquisition of FH signal respecting time of arrival and doppler search[7, 8], this paper focuses on the ionosphere's impact on the acquisition. The ionosphere dispersion can be expressed as a function of the TEC and the radio frequency of the signal as below

$$
H(f)=\frac{40.3}{c \cdot f^{2}} \cdot \mathrm{TEC} .
$$

Signal propagation path TEC is the product of vertical TEC and the cosecantof the elevation angle. Replacing the vertical TEC with an appropriate symbol $e_{\mathrm{TEC}}$, the ionosphere delay(in meter) of a radio wave with a frequency $f$ can be written as[9]

$$
\varepsilon^{I O N O}=\frac{1}{\sin \varphi} \cdot \frac{40.3}{f^{2}} \cdot e_{\mathrm{TEC}}
$$

$\varphi$ is the included angle between the ground and the satellite-ground-station connection(also known as the elevation angle), fis the radio frequency of the radio wave, $c$ is the velocity of light. $e_{\mathrm{TEC}}$ is mainly influenced by those factors including the sun radiation, the earth's magnetic field, and showing a decreasing 
trend from the equator to the north and south poles, and has a 24-hour-cycle, at about 2 p.m. local time, $e_{\text {TEC }}$ reaches a peak value. And in the earth's ionosphere $e_{\text {TEC }}$ ranges from $10^{16}$ to $10^{20}$ electrons $/ \mathrm{m}^{2}$.

The phase advance of the frequency hopping waveforms caused by ionosphere can be written as

$$
\theta_{i}=-\frac{2 \pi}{\sin \varphi} \cdot \frac{40.3}{c \cdot f_{i}} \cdot e_{\mathrm{TEC}}
$$

Taking (4) into consideration, equation (2) transforms into

$$
R_{i}^{\mathrm{IONO}}(v, \tau)=e^{j \theta_{i}} \int_{(i-1) T_{h}}^{i T_{h}} e^{-j 2 \pi\left[f_{i} \cdot \tau-\left(f_{R F}+f_{i}\right) \frac{(i-1) T_{h} v}{c}\right]} \mathrm{d} t .
$$

Because the hopping frequency hopping pattern is random, the closed form expression of the autocorrelation function can not be obtained. Thus replacing (5) into (1) and we obtain

$$
R^{\mathrm{IONO}}(v, \tau)=\sum_{i=1}^{N} R_{i}^{\mathrm{IONO}}(v, \tau)
$$

As mentioned in Introduction part, this paper mainly deals with the acquisition under ionosphere, so we assume that $v=0$ and $\tau=0$. According to (6), Figure 1 simulates the autocorrelation function $R^{\mathrm{IONO}}$ when $e_{\mathrm{TEC}}$, RF frequency, the satellite angle of view and frequency hopping bandwidth change. $e_{\mathrm{TEC}}$ ranges from $0 \sim 10^{20}$ electron $/ \mathrm{m}^{2}$, RF frequency increases from $1.5 \mathrm{GHz}$ to $2.5 \mathrm{GHz}$ with an interval of $0.1 \mathrm{GHz}$, satellite elevation angle increases from $10^{\circ}$ to $90^{\circ}$ with an interval of $10^{\circ}$ and the $\mathrm{FH}$ bandwidth is pick from the set $\{40 \mathrm{MHz}, 80 \mathrm{MHz}$, $120 \mathrm{MHz}, 160 \mathrm{MHz}, 200 \mathrm{MHz}$.

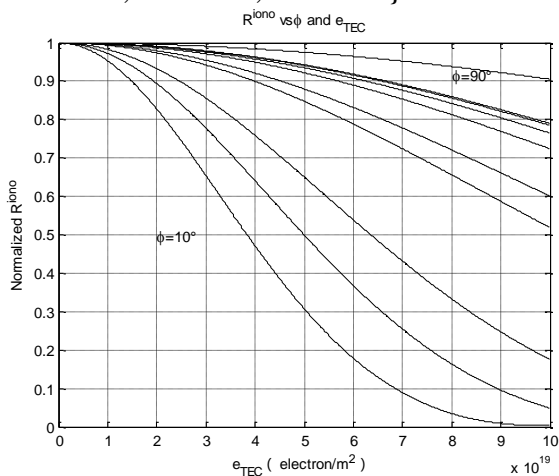

a. $\mathrm{R}^{\text {iono }}$ vs $e^{\mathrm{TEC}}$ and the elevation angle

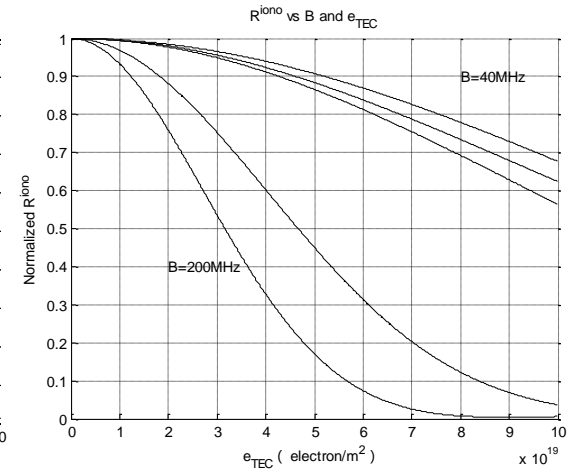

b. $\mathrm{R}^{\text {iono }}$ vs $e^{\mathrm{TEC}}$ and $\mathrm{FH}$ bandwidth

Fig.1.Autocorrelation function $\mathrm{R}^{\mathrm{iono}} \mathrm{vs} e_{\mathrm{TEC}}$, elevation angle, $\mathrm{FH}$ bandwidth and RF frequency

From Figure 1 it is referred that when the FH band is wider, the ionosphere dispersion is worse, when the elevation angle is lower, the dispersion is worse, when the radio frequency is lower, the dispersion is worse and when eTEC is 
larger, the dispersion is worse.If the $e_{\mathrm{TEC}}$ and the elevation angle $\varphi$ are known or confined to certain ranges, the ionosphere's impact on the acquisition can be eliminated.

\section{Ionosphere-Grid-Aided Fast Acquisition Algorithm}

The ionosphere-grid-aided fast acquisition(IGAFA) algorithm is presented in this section to deal with the fast acquisition of the phase coherent FFH signal mentioned in the last section. The core of the algorithm is the energy loss compensation based on ionosphere loss compensation method.Firstly here we introduce the basic signal processing structure in the acquisition of a phasecoherent fast FH signal. As shown in Figure 2the I/Q signals in intermediate frequency(IF) are de-hopped. Weassume that the search in time domain and Doppler frequency is finished and the integral in each hop is done. The TEC search algorithm in this paper is based on the ideal results of single hop integral.The presented IGAFA algorithm locates in the ionosphere dispersion compensation block in Figure 2.

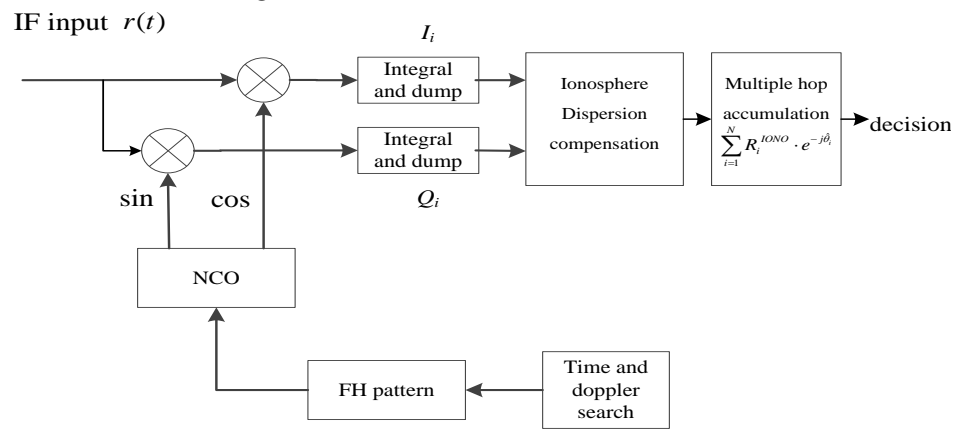

Fig.2. Signal acquisition of phase-coherent FFH signal

According to the spectrum resource assigned by international telecommunication union(ITU), the spectrum of this FFH signal is regulated as Table.1.

Table.1Signal parameters of the FFH signal

\begin{tabular}{lllll}
\hline FH band & RF frequency & TEC range & Symbol rate & FH rate \\
\hline $200 \mathrm{MHz}$ & $2.5 \mathrm{GHz}$ & $0 \sim 10^{20}$ electron $/ \mathrm{m}^{2}$ & $2 \mathrm{kBaud}$ & $40,000 \mathrm{hop} / \mathrm{s}$ \\
\hline
\end{tabular}

The principle of IGAFA algorithm is to use the $e_{\text {TEC }}$ provided by global ionosphere grid and the satellite elevation angle to calculate the phase advance caused by ionosphere transmission,perform coherent accumulation among different hops and compensate the energy loss. The ionosphere grid is mapped from the dual frequency navigation receiver of GNSS system such as GPS and Beidou System, the precision of $e_{\mathrm{TEC}}$ is around 2 to $10 \mathrm{TECU}$. Taking the lowest satellite elevation angle into consideration, like $10^{\circ}$, the worst error of $e_{\mathrm{TEC}}$ does 
not exceed $10 / \sin \left(10^{\circ}\right)=58 \times 10^{16}$ electron $/ \mathrm{m}^{2}$. From Figure 1 under the signal parameter in this paper, the acquisition energy loss caused by IonosphereGriderror does not exceed $0.5 \mathrm{~dB}$, so the IGAFA algorithm can guarantee the signal acquisition performance. The flow chart of IGAFA algorithm is shown in Figure 3 . Using the observation value of $e_{\mathrm{TEC}}$, the estimated phase advance is calculated as

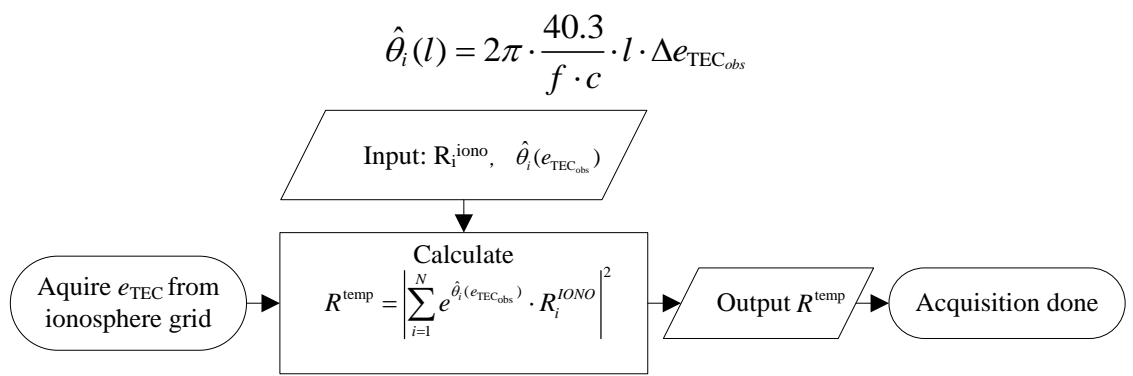

Fig.3. IGAFA algorithm flow chart

From Figure 3, the IGAFA algorithm does NOT need to search $e_{\mathrm{TEC}}$, instead it use the known global ionosphere grid information, thus the complexity remain $\mathrm{O}(\mathrm{n})$. To the FFH signal using the signal parameters in Table 1, the complexity of acquisition is reduced by

$$
L=T E C_{\text {Range }} / \triangle T E C=10^{20} / 2.2 \times 10^{20} \approx 5 \text { times. }
$$

\section{Computer Simulation}

These simulations are carried out usingMatlab. The simulation conditions are as follow. The signal sampling rate is $900 \mathrm{MHz}$, the $\mathrm{FH}$ bandwidth is $200 \mathrm{MH}$, the $\mathrm{RF}$ frequency is $2.5 \mathrm{GHz}, e_{\mathrm{TEC}}=5 \times 10^{19}$ electron $/ \mathrm{m}^{2}$, the symbol rate is $2 \mathrm{kBaud}$ the frequency hopping rate is $40,000 \mathrm{hop} / \mathrm{s}$, the length of $\mathrm{FH}$ sequence is $N=20$, the doppler frequency is zero and the symbol signal-to-noise ratio (SNR) ranges from $0 \mathrm{~dB}$ to $35 \mathrm{~dB}$ with the step of $1 \mathrm{~dB}$. The integration time length is $0.5 \mathrm{~ms}$, equal to the time length of one symbol. The CFAR is set as 0.001 . 


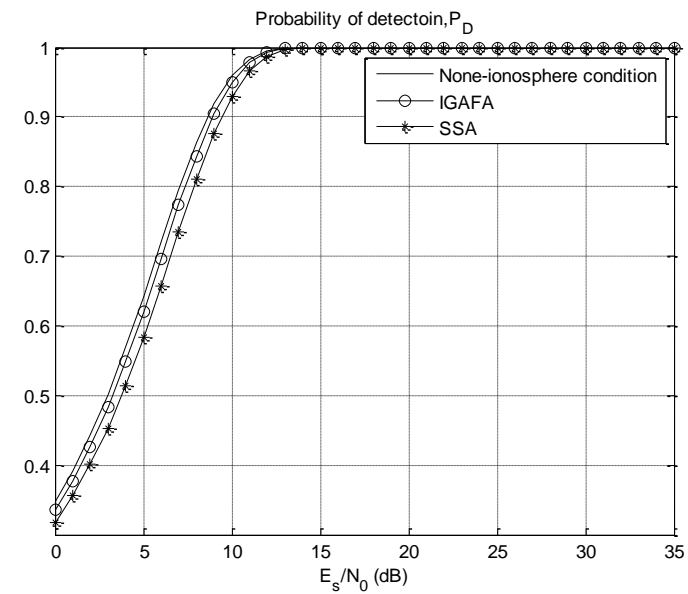

Fig.4. Probability of detection under different SNR(both IGAFA and SSA algorithms)

The simulation result in Figure 4 shows that 1)when the SNR in one single symbol $\left(E_{\mathrm{S}} / N_{0}\right)$ is larger than $14 \mathrm{~dB}$, both the traditional SSA algorithm and the IGAFA algorithm can approach $99 \%$ probability of detection(PD).2)The IGAFA algorithm not only lowered the searching complexity of $e_{\mathrm{TEC}}$, but also saved $1 \mathrm{~dB}$ energy loss when the PD is $99 \%$.

\section{Conclusion}

In dealing with the ionosphere's effect on FFH signal acquisition, this paper takes the lead in analyzing the ionosphere's impact on the coherent integration in signal acquisition, presents the IGAFA signal acquisition algorithm which exploits the ionosphere grid information and reduces the acquisition algorithm's complexity from $\mathrm{O}(\mathrm{Ln})$ to $\mathrm{O}(n)$. Comparing to traditional SSA algorithm which serially captures the $e_{\mathrm{TEC}}$, the IGAFA algorithm saves the acquisition time and in turn reduces the acquisition circuits design in the onboard receiver.

Acknowledgement

\section{Acknowledgments}

This work is supported by PLA Science Research Foundation (No. EP2015032200B21087).

\section{References:}

1. G. CHERUBINI, L.B. MILSTEIN, Performance analysis of both hybrid and frequency-hopped phase-coherent spread-spectrum systems. II. An FH system, Communications, IEEE Transactions on. 37,612-22(1989).

2. Y. HE, Y. CHENG, Y. YANG, G. WU, B. DONG, S. LI, A Subset-Based 
Coherent FFH System, IEEE COMMUN LETT. 19,199-202(2015).

3. M.K. SIMON, J.K. OMURA, R.A. SCHOLTZ, B.K. LEVITT, Spread spectrum communications handbook, (Citeseer1994).

4. E. KAPLAN, C. HEGARTY, Understanding GPS: principles and applications, (Artech house2005).

5. A.D. SARMA, D.V. RATNAM, D.K. REDDY, Modelling of low-latitude ionosphere using modified planar fit method for GAGAN, Radar, Sonar \& Navigation, IET. 3,609-19(2009).

6. G. CHERUBINI, L.B. MILSTEIN, Performance analysis of both hybrid and frequency-hopped phase-coherent spread-spectrum systems. I. A hybrid DS/FH system, Communications, IEEE Transactions on. 37,600-11(1989).

7. A. WEINBERG. Precise satellite ranging and timing system using pseudonoise bandwidth synthesis.: (Google Patents, 1991).

8. N. BENVENUTO, G. GUIDOTTI, S. PUPOLIN. Performance of a digital acquisition circuit for hybrid FH-DS spread spectrum systems. Military Communications Conference, 1988. MILCOM 88, Conference record. 21st Century Military Communications-What's Possible? 1988 IEEE.: (IEEE, 1988): 971-75.

9. K.C. YEH, C. LIU, Radio wave scintillations in the ionosphere, P IEEE. 70,324-60(1982). 